\title{
Particle-core analysis of dispersion effects on beam halo formation
}

\author{
Masanori Ikegami \\ Proton Accelerator Laboratory, Japan Atomic Energy Research Institute, Tokai-mura, Naka-gun, Ibaraki-ken 319-1195, Japan \\ Shinji Machida and Tomonori Uesugi* \\ Tanashi-branch, High Energy Accelerator Research Organization (KEK), Midori-cho, Tanashi-shi, Tokyo 188-8501, Japan
}

(Received 1 June 1999; published 13 December 1999)

\begin{abstract}
A simple particle-core model for circular accelerators has been constructed assuming that dispersion effects are relatively weak. This model is applicable to a large class of high-intensity rings designed for modest density applications such as spallation neutron sources. Applying this model to isotropic beams in a smooth ring, halo formation processes in the presence of dispersion are investigated. In the analysis, it is found that dispersion matching is essential to suppress horizontal beam widening in injection if momentum spread is larger than a certain threshold. Even if the beam widening due to dispersion mismatch is suppressed, a halo can be formed in the same mechanism as in a linac, namely, parametric resonance between oscillating core and single particles. The width of halos formed by the particlecore resonance in modest-density rings is found to be a little narrower than that in a straight channel, and typically 1.6 to 1.8 times the maximum core width.
\end{abstract}

PACS numbers: 41.75.-i, 29.27.Bd, 52.25.Wz

\section{INTRODUCTION}

Recently, high intensity ion beams have been proposed to be used for a wide variety of accelerator-related applications such as spallation neutron sources, transmutation of nuclear waste, heavy ion fusion, etc. In realizing these next generation high-intensity accelerators, beam loss minimization is essential to prevent excessive radioactivation of the accelerator. From this point of view, beam halo formation mechanism in a linac has been investigated extensively for the past several years. In these studies, parametric resonance between core and single particle oscillation is found to be a main cause of halo generation [1,2]. Furthermore, it has been gradually realized that halo formation with the same mechanism may happen and cause serious problems even in high-intensity circular accelerators where beam density is usually much lower than in linacs. In this context, increasing attention has recently been given to the beam halo problem in circular accelerators [3-5]. Extending the range of halo study into circular accelerators, we should take two additional effects into consideration. One is anisotropy of beams, or the difference of the emittance and external focusing field strength in two transverse directions. The other is the effect of longitudinal momentum spread in bending magnets.

In recent papers [6], Ikegami examined the effects of anisotropy on beam halo formation applying the particlecore model to anisotropic beams in a straight channel. The particle-core analysis [1] which is developed to

\footnotetext{
*Also at Department of Physics, University of Tokyo, Hongo, Bunkyo-ku, Tokyo 113-0033, Japan.
}

examine beam halo dynamics provides us a good physical insight into halo dynamics because of its simplicity. In that analysis, it was found that coupling between the horizontal and vertical coherent oscillations plays a key role in enhancing halo intensity, and that the coupling strength is strongly dependent on the degree of anisotropy. While these findings seem to provide valuable information for high-intensity ring design, effects of dispersion are not included in that study.

In some of the studies on beam halos in a ring, the emphasis is put upon the combined effect of space charge and dispersion [4,5]. Although the particle-core model was adopted in one of these studies [4], the multiparticle tracking approach was adopted instead of the Poincaré surface of section technique. As has been widely accepted, Poincaré surface of section technique has remarkably succeeded in deepening our understanding of underlying physics of halo formation processes in straight channels. Therefore, it is worth trying to construct an alternate particle-core model for circular accelerators which provides us a Poincaré surface of section plot of test particles, and to bring out the dispersion effects on halo formation processes. From that point of view, we concentrate in this paper on simplified situations rather than considering realistic lattice structures.

Another characteristic of beams in a ring is their modest beam density. Although there exists some exceptions, such as laser-cooler rings and the small electron ring at the University of Maryland [7] where beam density can be extremely high, beam density in high-intensity rings now operating or under proposal is usually much lower than in high-intensity linacs. For example, in designing proton synchrotrons or storage rings for spallation neutron sources, tune depression, or ratio of depressed tune to undepressed one, is usually chosen to be sufficiently 
larger than 0.9 to reduce space charge effects $[8,9]$. This beam density is much lower than that in their injector linacs where tune depression is typically around 0.6. Even with such modest beam density, uncontrollable beam losses due to halo formation is often considered as a main concern in designing high-intensity rings. Therefore, it is practically important to investigate halo dynamics in a ring especially with modest beam density and to estimate the halo width to determine the physical aperture of highintensity circular accelerators.

The purpose of this paper is to seek a possibility of constructing a simple particle-core model for beams in modest-density circular accelerators and to clearly understand the effects of dispersion on halo formation in these machines. In this paper, we consider the simplest situation to bring out the dispersion effect on halo dynamics, namely, a coasting beam circulating in a smooth ring where both the bending and focusing fields are constant (smooth approximation). The emphasis is put upon situations with modest beam density where tune depression is around or larger than 0.9 , while halo formation in higher density cases is also discussed. To be noted here is that it is a reasonable approximation to consider a coasting beam because synchrotron tune is generally much smaller than betatron tune in a ring, and, hence, coherent behavior of a beam is well described by the two dimensional envelope equations [10].

This paper is organized as follows: Dispersion effects on both core and test particle evolution are examined in Sec. II. Based on the findings in this section, we develop a simple particle-core model for beams in circular accelerators in Sec. III, and apply it to isotropic beams in a smooth ring in Sec. IV. Then, a summary is given in the last section.

\section{EFFECT OF DISPERSION}

\section{A. Envelope equation with dispersion}

The first basis of the particle-core analysis is envelope equations which describe the time evolution of the core envelope. Recently, envelope equations which include effects of dispersion and space charge were derived by Venturini and Reiser [11]. They found that the usual horizontal rms emittance $\epsilon_{x}^{2}=\left\langle x^{2}\right\rangle\left\langle p_{x}^{2}\right\rangle-\left\langle x p_{x}\right\rangle^{2}$ is not an invariant of motion in the presence of dispersion. Here, $\langle X\rangle$ denotes the ensemble average of $X$ over the phase space distribution. Instead, there exists a new invariant $\epsilon_{d x}$ which can be written as

$$
\begin{aligned}
\epsilon_{d x}^{2}= & \left(\left\langle x^{2}\right\rangle-D^{2} \Delta^{2}\right)\left(\left\langle p_{x}^{2}\right\rangle-D^{\prime 2} \Delta^{2}\right) \\
& -\left(\left\langle x p_{x}\right\rangle-D D^{\prime} \Delta^{2}\right)^{2},
\end{aligned}
$$

where $\Delta=\sqrt{\left\langle(\delta p / p)^{2}\right\rangle}$ is rms momentum spread. The prime notation refers to differentiation with respect to the longitudinal distance $s$. The dispersion function $D$ obeys the equation

$$
D^{\prime \prime}+\left[\kappa_{x}-\frac{K}{2 a(a+b)}\right] D=\frac{1}{\rho},
$$

where $a=\sqrt{\left\langle x^{2}\right\rangle}$ and $b=\sqrt{\left\langle y^{2}\right\rangle}$ are rms beam widths, and $\rho$ is the average radius of curvature. The generalized perveance $K$ is a measure of beam density and $\kappa_{x}$ is a constant which represents external focusing field strength in the horizontal direction. Note here that $\kappa_{x}$ includes the horizontal focusing effect of bending magnets. In the following, we will refer to $\epsilon_{d x}$ as generalized emittance.

The envelope equations with dispersion can be written as

$$
\begin{aligned}
& a^{\prime \prime}+\kappa_{x} a-\frac{K}{2(a+b)}-\frac{\Delta^{2}}{a}\left(\frac{D}{\rho}+D^{\prime 2}\right)- \\
& \frac{\epsilon_{d x}^{2}-a^{\prime 2}\left(a^{2}-D^{2} \Delta^{2}\right)+\left(a a^{\prime}-D D^{\prime} \Delta^{2}\right)^{2}}{a\left(a^{2}-D^{2} \Delta^{2}\right)}=0,
\end{aligned}
$$

and

$$
b^{\prime \prime}+\kappa_{y} b-\frac{K}{2(a+b)}-\frac{\epsilon_{y}^{2}}{b^{3}}=0,
$$

where $\epsilon_{y}$ and $\kappa_{y}$ are, respectively, rms emittance and external focusing field strength in the vertical direction. As easily seen in Eq. (3), the emittance term is modified by adopting the generalized emittance, and the dispersion term is added compared to envelope equations for a straight channel. Simultaneously solving Eqs. (2)-(4), we can obtain the time evolution of the core envelope.

To construct a particle-core model for beams with finite momentum spread, we need to know the matched beam width and coherent tune of these beams. As easily seen in Eqs. (2) -(4), the presence of dispersion causes changes of matched beam size and coherent tune. In the following subsections, we estimate these changes due to dispersion considering isotropic situations where $\kappa_{x}=\kappa_{y}$ and $\epsilon_{x}=\epsilon_{y}$.

\section{B. Matched beam width}

Here we consider the matched situation where $a^{\prime}=$ $b^{\prime}=D^{\prime}=0$. First, let us start with a trivial case where there exists bending field but beams have no momentum spread, $\Delta=0$. In this case, the envelope equations reduce to a usual set of equations for straight channels, and its matched beam width $\hat{a}_{0}$ is easily obtained by solving

$$
\kappa_{x} \hat{a}_{0}-\frac{K}{4 \hat{a}_{0}}-\frac{\epsilon_{x}^{2}}{\hat{a}_{0}^{3}}=0,
$$

where $\epsilon_{x}$ is constant.

Proceeding to cases with finite momentum spread, we consider two beams which have the same emittance, generalized perveance and bare tune, but different momentum spread; namely, one has finite momentum spread but the 
other has no momentum spread. Because of dispersion effects, the matched beam width and coherent tune of these two beams are generally different. In this subsection, we try to obtain the difference between matched beam width of a beam with finite momentum spread and that of its corresponding zero-momentum-spread beam.

The matched solutions $a_{0}, b_{0}$, and $D_{0}$ for beams with finite momentum spread can be obtained by solving

$$
\begin{gathered}
{\left[\kappa_{x}-\frac{K}{2 a_{0}\left(a_{0}+b_{0}\right)}\right] D_{0}=\frac{1}{\rho},} \\
\kappa_{x} a_{0}-\frac{K}{2\left(a_{0}+b_{0}\right)}-\frac{\Delta^{2}}{a_{0}} \frac{D_{0}}{\rho}-\frac{\epsilon_{x}^{2}}{a_{0}^{3}}=0,
\end{gathered}
$$

and

$$
\kappa_{x} b_{0}-\frac{K}{2\left(a_{0}+b_{0}\right)}-\frac{\epsilon_{x}^{2}}{b_{0}^{3}}=0 .
$$

To be noted here is that the horizontal emittance $\epsilon_{x}$ is conserved in matched situations even in the presence of dispersion.

Considering situations where dispersion effect is modest, we here denote the matched solutions as

$$
a_{0}=\hat{a}_{0}\left(1+\delta_{a 0}\right),
$$

and

$$
b_{0}=\hat{a}_{0}\left(1+\delta_{b 0}\right),
$$

where $\hat{a}_{0}$ is the matched beam width of the corresponding zero-momentum-spread beam. Substituting them into Eqs. (7) and (8) and dropping second or higher order terms of $\delta_{a 0}$ and $\delta_{b 0}$, we obtain

$$
\delta_{a 0}=\frac{\hat{\eta}_{x}^{2}\left(3+5 \hat{\eta}_{x}^{2}\right)}{4\left(1+\hat{\eta}_{x}^{2}\right)\left(1+3 \hat{\eta}_{x}^{2}\right)} \hat{\xi}^{2},
$$

and

$$
\delta_{b 0}=-\frac{\hat{\eta}_{x}^{2}\left(1-\hat{\eta}_{x}^{2}\right)}{4\left(1+\hat{\eta}_{x}^{2}\right)\left(1+3 \hat{\eta}_{x}^{2}\right)} \hat{\xi}^{2},
$$

where $\hat{\eta}_{x}$ is the tune depression for the corresponding zero-momentum-spread beam. The parameter $\hat{\xi}$ is a measure of the strength of dispersion effect defined by

$$
\hat{\xi}=\frac{\Delta}{\hat{a}_{0} \rho \hat{\eta}_{x}^{2} \kappa_{x}} .
$$

As $\hat{\xi}^{2}$ is typically several percent in modest-density circular accelerators, as discussed later, fourth or higher order terms of $\hat{\xi}$ are neglected in the derivation of Eqs. (11) and (12).

It is worth noting here that the presence of dispersion gives slight anisotropy to beams as

$$
\frac{b_{0}}{a_{0}}=1-\frac{\hat{\eta}_{x}^{2}}{1+3 \hat{\eta}_{x}^{2}} \hat{\xi}^{2}
$$

The change of matched beam width also causes a difference of tune depression. The horizontal tune depression $\eta_{x}$ in the presence of dispersion can be written as

$$
\eta_{x}^{2}=\left[1+\frac{\left(1-\hat{\eta}_{x}^{2}\right)\left(1+2 \hat{\eta}_{x}^{2}\right)}{\left(1+\hat{\eta}_{x}^{2}\right)\left(1+3 \hat{\eta}_{x}^{2}\right)} \hat{\xi}^{2}\right] \hat{\eta}_{x}^{2} .
$$

\section{Coherent tune}

In mismatched situations, core envelope and dispersion function oscillate around the matched solution. Considering weakly mismatched cases, we here approximate the oscillation as

$$
\begin{aligned}
& a=a_{0}\left[1+\delta_{a}(s)\right], \\
& b=b_{0}\left[1+\delta_{b}(s)\right],
\end{aligned}
$$

and

$$
D=D_{0}\left[1+\delta_{D}(s)\right],
$$

where $\delta_{a}(s), \delta_{b}(s)$, and $\delta_{D}(s)$ are periodic functions of $s$ with small amplitude. In contrast to matched cases, the horizontal emittance $\epsilon_{x}$ also oscillates around the matched horizontal emittance $\epsilon_{0}$, which can be written as

$$
\epsilon_{0}^{2}=\epsilon_{d x}^{2}+a_{0}^{4} \eta_{x}^{2} \kappa_{x} \xi^{2},
$$

where $\xi$ is another measure of the strength of dispersion effect defined in an analogous way with $\hat{\xi}$ as

$$
\xi=\frac{\Delta}{a_{0} \rho \eta_{x}^{2} \kappa_{x}} .
$$

With the use of Eqs. (11) and (15), the relation between $\hat{\xi}$ and $\xi$ can be written as

$$
\xi=\left[1-\frac{4+7 \hat{\eta}_{x}^{2}-3 \hat{\eta}_{x}^{4}}{4\left(1+\hat{\eta}_{x}^{2}\right)\left(1+3 \hat{\eta}_{x}^{2}\right)} \hat{\xi}^{2}\right] \hat{\xi} .
$$

Neglecting second or higher order terms of $\delta_{a}(s)$, $\delta_{b}(s)$, and $\delta_{D}(s)$, the time evolution of the emittance is obtained as

$$
\begin{aligned}
\epsilon_{x}^{2}= & \epsilon_{0}^{2}+\frac{1}{2} a_{0}^{4}\left(3+\eta_{x}^{2}\right) \kappa_{x} \xi^{2} \delta_{a}(s) \\
& +\frac{1}{2} a_{0}^{4}\left(1-\eta_{x}^{2}\right) \kappa_{x} \xi^{2} \delta_{b}(s)+2 a_{0}^{4} \eta_{x}^{2} \kappa_{x} \xi^{2} \delta_{D}(s)
\end{aligned}
$$

In the derivation of Eq. (22), we neglect fourth or higher order terms of $\xi$ also.

Substituting these equations into Eq. (2)-(4), we obtain a set of equations which describe the time evolution of $\delta_{a}(s), \delta_{b}(s)$, and $\delta_{D}(s)$. Neglecting higher order terms, 
the set of equations reduces to

$$
\begin{gathered}
\delta_{a}^{\prime \prime}(s)+\left[\frac{3+5 \eta_{x}^{2}}{2}-\frac{6+27 \eta_{x}^{2}+31 \eta_{x}^{4}}{4\left(1+3 \eta_{x}^{2}\right)} \xi^{2}\right] \kappa_{x} \delta_{a}(s)+ \\
{\left[\frac{1-\eta_{x}^{2}}{2}-\frac{\left(1-\eta_{x}^{2}\right)\left(2+7 \eta_{x}^{2}\right)}{4\left(1+3 \eta_{x}^{2}\right)} \xi^{2}\right] \kappa_{x} \delta_{b}(s)-3 \eta_{x}^{2} \xi^{2} \kappa_{x} \delta_{D}(s)=0,} \\
\delta_{b}^{\prime \prime}(s)+\left[\frac{1-\eta_{x}^{2}}{2}+\frac{\eta_{x}^{2}\left(1-\eta_{x}^{2}\right)}{4\left(1+3 \eta_{x}^{2}\right)} \xi^{2}\right] \kappa_{x} \delta_{a}(s)+\left[\frac{3+5 \eta_{x}^{2}}{2}-\frac{\eta_{x}^{2}\left(17-\eta_{x}^{2}\right)}{4\left(1+3 \eta_{x}^{2}\right)} \xi^{2}\right] \kappa_{x} \delta_{b}(s)=0,
\end{gathered}
$$

and

$$
\delta_{D}^{\prime \prime}(s)+\left[\frac{3\left(1-\eta_{x}^{2}\right)}{2}+\frac{\eta_{x}^{2}\left(1-\eta_{x}^{2}\right)}{4\left(1+3 \eta_{x}^{2}\right)} \xi^{2}\right] \kappa_{x} \delta_{a}(s)+\left[\frac{1-\eta_{x}^{2}}{2}-\frac{\eta_{x}^{2}\left(1-\eta_{x}^{2}\right)}{4\left(1+3 \eta_{x}^{2}\right)} \xi^{2}\right] \kappa_{x} \delta_{b}(s)+\eta_{x}^{2} \kappa_{x} \delta_{D}(s)=0 .
$$

The general solution of Eqs. (23)-(25) is a superposition of three normal modes which we will refer to as breathing, quadrupole, and dispersion modes. In the zerocurrent limit, coupling between transverse beam widths and dispersion function obviously vanishes and the oscillation of dispersion function is dominated by a single mode which we refer to as the dispersion mode. On the other hand, the oscillation of transverse beam widths becomes a superposition of two normal modes. One is the breathing mode in which $a$ and $b$ oscillate in phase, and the other is the quadrupole mode in which $a$ and $b$ oscillate $180^{\circ}$ out of phase. In the presence of space charge, oscillations of beam widths and dispersion function are coupled and the general solution can be written as

$$
\begin{aligned}
& \delta_{a}(s)=C_{11} \Psi_{b}(s)+C_{21} \Psi_{q}(s)+C_{31} \Psi_{d}(s), \\
& \delta_{b}(s)=C_{12} \Psi_{b}(s)+C_{22} \Psi_{q}(s)+C_{32} \Psi_{d}(s),
\end{aligned}
$$

and

$$
\delta_{D}(s)=C_{13} \Psi_{b}(s)+C_{23} \Psi_{q}(s)+C_{33} \Psi_{d}(s),
$$

where $\Psi_{b}(s), \Psi_{q}(s)$, and $\Psi_{d}(s)$ are eigenfunctions which, respectively, correspond to breathing, quadrupole, and dispersion modes. The eigenfunctions can be written as

$$
\begin{aligned}
& \Psi_{b}(s)=\exp \left[\frac{i\left(\nu_{b}-\varphi_{1}\right) s}{\rho}\right], \\
& \Psi_{q}(s)=\exp \left[\frac{i\left(\nu_{q}-\varphi_{2}\right) s}{\rho}\right],
\end{aligned}
$$

and

$$
\Psi_{d}(s)=\exp \left[\frac{i\left(\nu_{d}-\varphi_{3}\right) s}{\rho}\right],
$$

where $\varphi_{1}$ to $\varphi_{3}$ are constants which depend on the initial condition, and $\nu_{b}, \nu_{q}$, and $\nu_{d}$ are, respectively, tunes of breathing, quadrupole, and dispersion modes of core oscillation. From Eqs. (23)-(25), these coherent tunes can be obtained as

$\nu_{b}^{2}=\left[2\left(1+\eta_{x}^{2}\right)-\frac{2+14 \eta_{x}^{2}+15 \eta_{x}^{4}-7 \eta_{x}^{6}}{\left(2+\eta_{x}^{2}\right)\left(1+3 \eta_{x}^{2}\right)} \xi^{2}\right] \nu_{x}^{2}$,

$$
\nu_{q}^{2}=\left[1+3 \eta_{x}^{2}-\frac{1+14 \eta_{x}^{2}+30 \eta_{x}^{4}+3 \eta_{x}^{6}}{2\left(1+2 \eta_{x}^{2}\right)\left(1+3 \eta_{x}^{2}\right)} \xi^{2}\right] \nu_{x}^{2}
$$

and

$$
\nu_{d}^{2}=\left[\eta_{x}^{2}+\frac{3 \eta_{x}^{2}\left(1-\eta_{x}^{2}\right)\left(4+5 \eta_{x}^{2}\right)}{2\left(2+\eta_{x}^{2}\right)\left(1+2 \eta_{x}^{2}\right)} \xi^{2}\right] \nu_{x}^{2}
$$

where $\nu_{x}=\sqrt{\kappa_{x}} \rho$ is the horizontal bare tune.

Relative oscillation amplitude in pure breathing, quadrupole, and dispersion oscillation cases are, respectively, shown in Figs. 1-3. As seen in these figures, in modest density cases, the oscillations of beam envelope $a$ and $b$ are nearly dominated by the breathing and quadrupole modes, while that of dispersion function $D$ is dominated by the dispersion mode as expected. In the breathing and quadrupole oscillation cases, the asymmetry in the two transverse directions is increased as $\xi$ is increased, while the symmetry is restored as beam density is increased.

\section{Test-particle equation of motion with dispersion}

The second basis of the particle-core analysis is the equation of motion of test particles. Assuming that cores have the KV distribution [12] in the transverse phase space and that test particles have zero angular momentum, the equation of motion for test particles initially located on the horizontal plane $\left(y=y^{\prime}=0\right)$ can be written as [13]

$$
x^{\prime \prime}+\kappa_{x} x-\frac{K}{2 a(a+b)} x-\frac{1}{\rho} \frac{\delta p}{p}=0,
$$

inside the core $(|x| \leq 2 a)$ and

$$
\begin{aligned}
& x^{\prime \prime}+\kappa_{x} x-\frac{K}{x^{2}+|x| \sqrt{x^{2}+4\left(b^{2}-a^{2}\right)}} x- \\
& \frac{1}{\rho} \frac{\delta p}{p}=0,
\end{aligned}
$$

outside the core $(|x|>2 a)$. Assuming the absence of acceleration or deceleration forces, the momentum deviation $\delta p / p$ is a particle-dependent constant. 

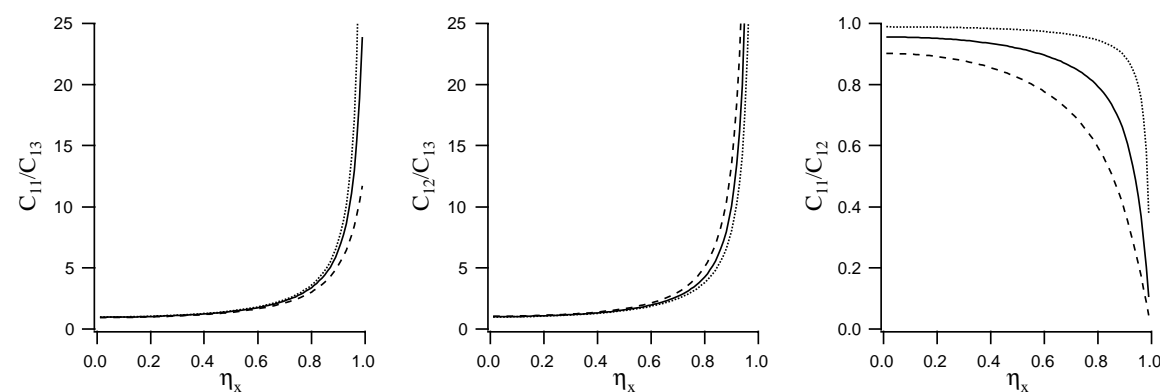

FIG. 1. The relative oscillation amplitude in breathing oscillation cases. Three different $\xi$ have been considered, i.e., $\xi=0.075$ (dotted line), $\xi=0.15$ (solid line); and $\xi=0.225$ (broken line).
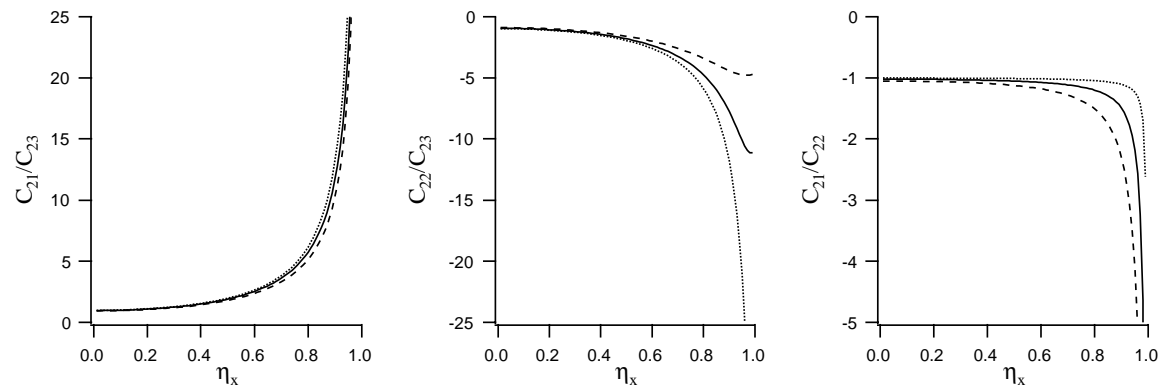

FIG. 2. The relative oscillation amplitude in quadrupole oscillation cases. Three different $\xi$ have been considered, i.e., $\xi=0.075$ (dotted line), $\xi=0.15$ (solid line), and $\xi=0.225$ (broken line).
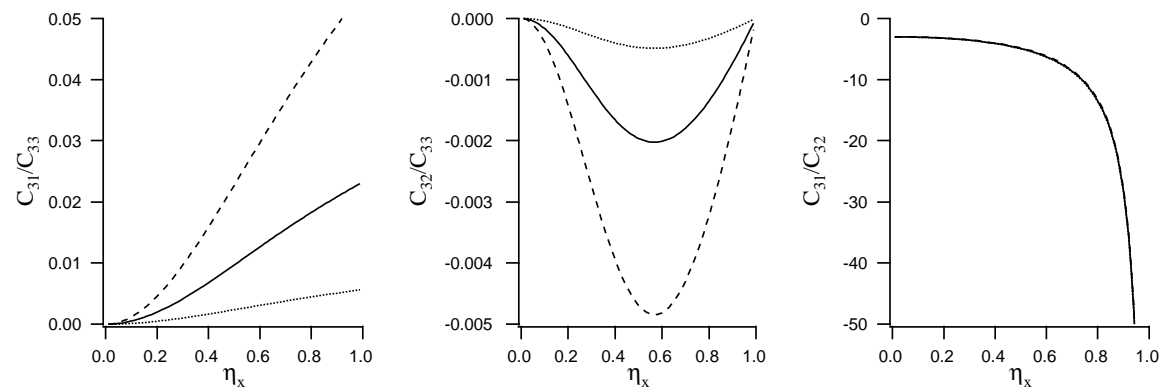

FIG. 3. The relative oscillation amplitude in dispersion oscillation cases. Three different $\xi$ have been considered, i.e., $\xi=0.075$ (dotted line), $\xi=0.15$ (solid line), and $\xi=0.225$ (broken line).

As easily seen in Eqs. (35) and (36), the effect of dispersion on test-particle motion is in the order of $\xi$. For example, the dispersion term in Eq. (35) provides a shift of minimal point of total potential field felt by the test particle. The amount of the shift scaled by the matched rms beam width $a_{0}$ is $\Lambda \xi$, where relative momentum deviation $\Lambda=(\delta p / p) / \Delta$ is a particle-dependent constant. The maximum value of $\Lambda$, which we denote $\Lambda_{\max }$ hereafter, typically ranges from two to three, while it is distribution dependent.

\section{E. Dispersion effect in typical rings}

In this subsection, we examine typical values of $\xi$ in circular accelerators designed for modest-density applica- tions. To obtain $\xi$ of an actual accelerator, we rewrite Eq. (20) into a more convenient form as

$$
\xi^{2}=\frac{\rho \Delta^{2}}{\epsilon_{0}\left(\eta_{x} \nu_{x}\right)^{3}} .
$$

As an example, we take parameters of proton storage rings for the Neutron Science Project (NSP), which has been proposed by Japan Atomic Energy Research Institute [14]. In NSP, a proton storage ring with 20 FODO cells is proposed as an option to realize bunch compression for spallation neutron sources. The main parameters of this ring are $|\delta p / p|<4 \times 10^{-3}$, $\rho=29.5 \mathrm{~m}, \epsilon_{100 \%}=2 \times 10^{-4} \mathrm{mrad}, \nu_{x}=4.84$, and $\eta_{x} \sim 0.98$, where $\epsilon_{100 \%}$ is full emittance in the horizontal 
direction. In obtaining $\xi$, we need to know $\Lambda_{\max }$ and $\epsilon_{100 \%} / \epsilon_{0}$ which are distribution-dependent constants. Assuming $2<\Lambda_{\max }<3$ and $4<\epsilon_{100 \%} / \epsilon_{0}<9$, we find $4.4 \times 10^{-3}<\xi^{2}<2.2 \times 10^{-2}$, which is small enough to justify the negligence of fourth or higher order terms of $\xi$. However, we should keep in mind that $\xi$ is strongly dependent on the choice of design parameters even if beam density is modest. In fact, in the triple bend achromat (TBA) option for NSP, $\xi$ is much larger than in the FODO option and $2.5 \times 10^{-2}<\xi^{2}<0.13$ because of its relatively weak focusing nature. Although it is dependent on the lattice structure and momentum acceptance, $\xi^{2}$ in circular accelerators for spallation neutron sources is generally around or smaller than 0.1 . Therefore, the negligence of fourth or higher order terms of $\xi$ is sufficient, at least, as the first stage for estimation of dispersion effects on halo formation in modest-density circular accelerators.

\section{PARTICLE-CORE MODEL}

The above analysis justifies the negligence of fourth or higher order terms of $\xi$ in halo studies for modestdensity circular accelerators, which enables us to construct a simple particle-core model for beams in these machines. Hereafter, we concentrate on pure breathing oscillation cases, as extending to quadrupole oscillation cases is straightforward. Assuming breathing oscillation cases $\left(C_{21}=C_{31}=0\right)$, envelope oscillation can be written as

$$
\tilde{a}=\tilde{a}_{0}\left[1+\mu_{x} \cos \left(\frac{\nu_{b}}{\nu_{x}} \tau\right)\right],
$$

and

$$
\tilde{b}=\tilde{b}_{0}\left[1+\mu_{y} \cos \left(\frac{\nu_{b}}{\nu_{x}} \tau\right)\right]
$$

where we introduce scaled variables $\tilde{a}=\sqrt{\nu_{x} / \rho \epsilon_{0}} a$, $\tilde{b}=\sqrt{\nu_{x} / \rho \epsilon_{0}} b, \quad \tilde{a}_{0}=\sqrt{\nu_{x} / \rho \epsilon_{0}} a_{0}, \quad$ and $\quad \tilde{b}_{0}=$ $\sqrt{\nu_{x} / \rho \epsilon_{0}} b_{0}$. The independent variable is taken as $\tau=\nu_{x} s / \rho$. Mismatch factors $\mu_{x}$ and $\mu_{y}$ are defined by $\mu_{x}=\left[a(0)-a_{0}\right] / a_{0}$ and $\mu_{y}=\left(C_{12} / C_{11}\right) \mu_{x}$, respectively. For later reference, we here introduce another measure of the degree of initial beam size mismatch $M$, which is defined by

$$
M=\sqrt{\frac{\mu_{x}^{2}+\mu_{y}^{2}}{2}} .
$$

Introducing similar scaled variables, the equation of motion for test particles initially located on the horizontal plane can be rewritten as

$$
\frac{d^{2} \tilde{x}}{d \tau^{2}}+\tilde{x}-\frac{\tilde{K}}{2 \tilde{a}(\tilde{a}+\tilde{b})} \tilde{x}-\eta_{x}^{3 / 2} \Lambda \xi=0,
$$

inside the core $(|\tilde{x}| \leq 2 \tilde{a})$ and

$$
\begin{aligned}
\frac{d^{2} \tilde{x}}{d \tau^{2}}+\tilde{x}-\frac{\tilde{K}}{\tilde{x}^{2}+|\tilde{x}| \sqrt{\tilde{x}^{2}+4\left(\tilde{b}^{2}-\tilde{a}^{2}\right)}} \tilde{x}- & \\
\eta_{x}^{3 / 2} \Lambda \xi=0, & \text {. }
\end{aligned}
$$

outside the core $(|\tilde{x}|>2 \tilde{a})$, where $\tilde{x}=\sqrt{\nu_{x} / \rho \epsilon_{0}} x$ and $\tilde{K}=\rho K /\left(\epsilon_{0} \nu_{x}\right)$.

Test particle evolution is obtained by simultaneously solving Eqs. (41) and (42) with Eqs. (38) and (39). In the integration, we employ the fourth-order symplectic integration algorithm [15]. In the rest of this paper, we omit tilde for brevity.

\section{NUMERICAL RESULTS}

\section{A. Modest density cases}

Applying the particle-core model introduced in the preceding section, we examine halo dynamics in the presence of dispersion. To start with, we consider a modest density beam with $\eta_{x}=0.9$ and $\xi=0.15$. The Poincaré surface of section plots for test particles is shown in Fig. 4. In this figure, test particles with three different $\Lambda$ have been considered, while the mismatch factor $M$ is fixed to 0.13. As seen in Fig. 4(a), the width of a 2:1 resonance island is a little narrower than that of the corresponding zero-momentum spread beam because of a decrease of coherent tune. For particles with momentum deviation, dispersion effect gives a shift of fixed point locations, and the width of a 2:1 resonance island decreases as $\Lambda$ is increased. As readily seen in Fig. 4, dispersion matching is essential if the maximum value of $\Lambda$ is larger than a certain threshold; namely, particles with large momentum must be injected into the outer side of the core and those with small momentum into the inner side. Without dispersion matching, the shift of fixed point locations provides a transport mechanism by which some of the particles initially located inside the core can escape from the core and become halos. To make clear the diffusion effect due to dispersion mismatch, the maximum horizontal extent reached by the particles initially located at $x=-2 a_{\max }$ and $d x / d \tau=0$ is shown in Fig. 5. The initial position corresponds to the inner edge of a mismatched core. Without dispersion matching, particles with positive momentum deviation can be injected into the inner side of the core. If $\Lambda$ is small enough, the test particles are kept orbiting in the vicinity of the core boundary, and, hence, dispersion mismatch only gives slight widening of the horizontal beam width. However, as $\Lambda$ is increased, these particles are trapped by the 2:1 resonance at a certain threshold $\Lambda=\Lambda_{0}$, and it enlarges oscillation amplitude significantly. In the case with $\eta_{x}=0.9$ and $\xi=0.15, \Lambda_{0}$ is around 2.1, as clearly seen in Fig. 3, and it varies roughly inversely as $\xi$ while its dependence on $M$ is weak. If $\Lambda$ is significantly larger 

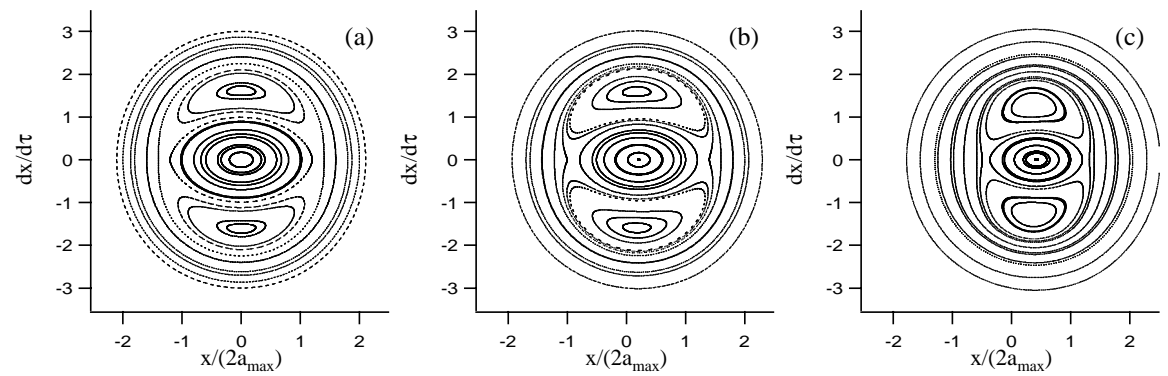

FIG. 4. The Poincaré surface of section plots for test particles in modest density cases. The test particle position scaled by the maximum beam boundary width $2 a_{\max }=2 a(0)$ is taken as the abscissa. Three different $\Lambda$, i.e., (a) $\Lambda=0$, (b) $\Lambda=2$, and (c) $\Lambda=4$, are considered. Other parameters are fixed to $\eta_{x}=0.9, M=0.13$, and $\xi=0.15$.

than the threshold $\Lambda_{0}$, these particles become off the resonance, but it does not result in a decrease of oscillation amplitude. The particles with too large $\Lambda$ start to orbit outside the separatrix of the 2:1 particle-core resonance. In short, diffusion due to dispersion mismatch makes it possible for some of the particles initially located inside the core to become halos if $\Lambda_{\max }$ is larger than $\Lambda_{0}$, and the width of the halos formed by this mechanism is not limited by the separatrix of the 2:1 particle-core resonance.

Even if the diffusion due to dispersion mismatch is avoided, halos can be formed by the same mechanism as in a straight channel, namely, resonant interaction between core and single particles. In this case, the trajectory of particles initially located in the vicinity of the core is bounded by the separatrix of the $2: 1$ particle-core resonance. Therefore, halo width can be estimated by the same way as for a straight channel, namely, plotting Poincaré plots with adequate strobe time and examining separatrix locations. The horizontal separatrix width is plotted in Fig. 6 as a function of $\Lambda$. It is readily seen in Fig. 6 that the separatrix width is generally a little narrower than that in a straight channel, while it depends on $\Lambda$. In the case with $\eta_{x}=0.9$ and $\xi^{2} \sim 0.1$, the maximum value of the separatrix width is found to be about 1.8 times the maximum core width, which is weakly dependent on $M$ and $\xi$.

The corresponding figures for lower density cases are shown in Figs. 7 and 8, where three different beam densities have been considered to examine the density dependence of the halo extent in the lower density region. In Fig. 7 , it is clearly seen that $\Lambda_{0}$ becomes smaller with lower beam density, which imposes more strict requirements for dispersion matching for lower density cases. If the diffusion due to dispersion mismatch is avoided, halos are expected to be formed by the 2:1 particle-core resonance. We can see in Fig. 8 that the width of halos formed by the particle-core resonance has weak dependence on beam density, and it is smaller with lower beam density. In fact, the maximum value of separatrix width is found to be about 1.6 times the maximum core width in the case with $\eta_{x}=0.98, M=$ 0.13 , and $\xi=0.15$. These results lead us to conclude that the dispersion matching is essential to avoid the diffusion in the NSP storage ring in both the FODO and TBA options. The halo width with sufficient dispersion matching is expected to be about $20 \%$ smaller than that estimated in a straight channel. While the current design of the NSP storage ring meets the requirement for the physical aperture, more optimization of lattice design may be preferable to achieve larger aperture and reduce the possibility of uncontrollable beam loss.

Summarizing the results shown in Figs. 5-8, halo width in a typical modest-density ring is about 1.6 to 1.8 times the maximum core width if $\Lambda_{\max } / \Lambda_{0}<1$, while it can be larger if $\Lambda_{\max } / \Lambda_{0}>1$. In the case of $\Lambda_{\max } / \Lambda_{0}>$ 1 , dispersion matching is essential to reduce both halo
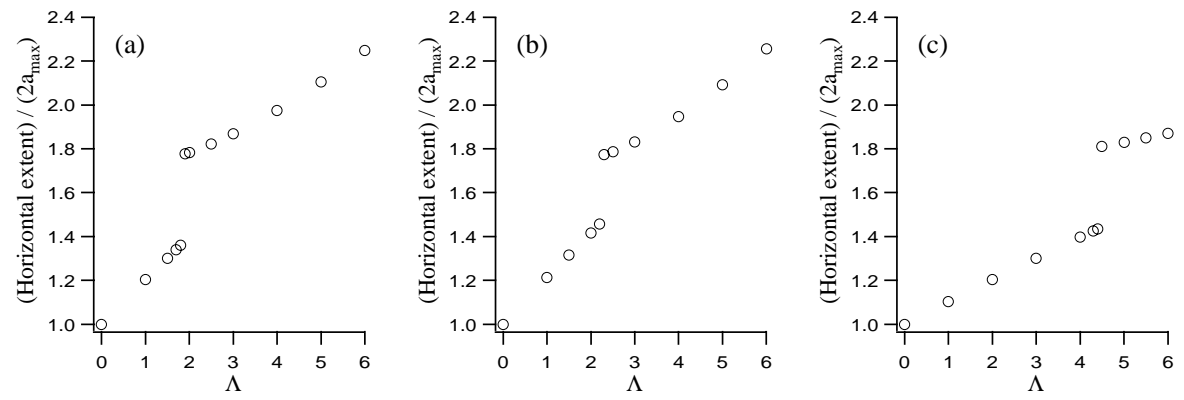

FIG. 5. The horizontal extent of the particle initially located at the inner edge of the core in the cases with $\eta_{x}=0.9$. Three different combinations of $M$ and $\xi$ are considered, i.e., (a) $M=0.13$ and $\xi=0.15$, (b) $M=0.066$ and $\xi=0.15$, and (c) $M=0.13$ and $\xi=0.075$. 

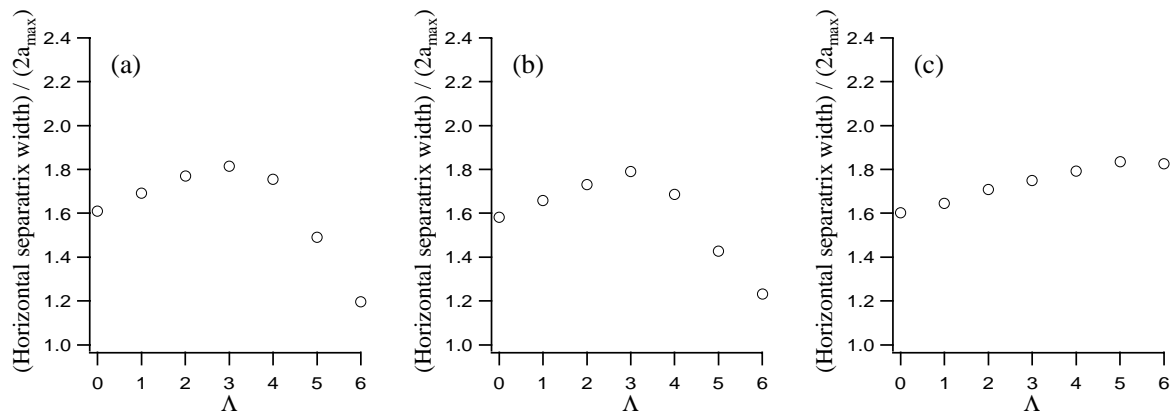

FIG. 6. The horizontal separatrix width of the $2: 1$ particle-core resonance in the cases with $\eta_{x}=0.9$. Three different combinations of $M$ and $\xi$ are considered, i.e., (a) $M=0.13$ and $\xi=0.15$, (b) $M=0.066$ and $\xi=0.15$, and (c) $M=0.13$ and $\xi=0.075$.
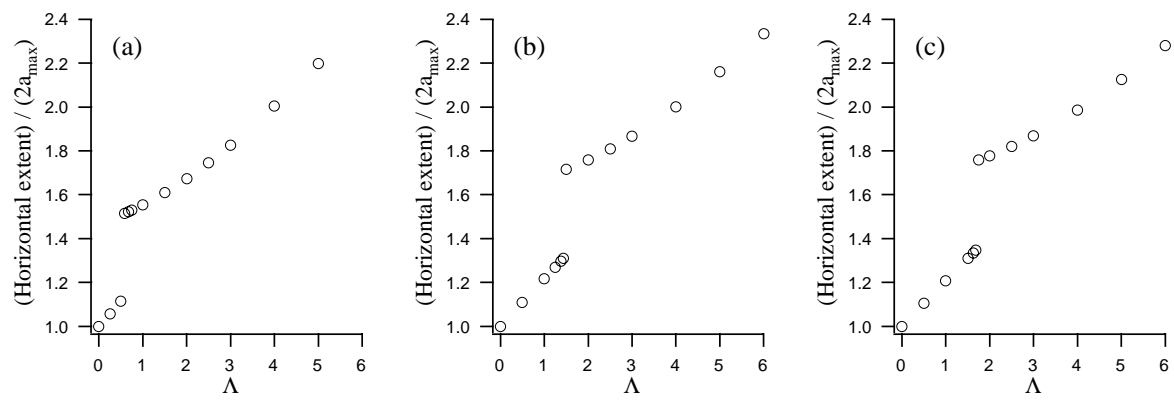

FIG. 7. The horizontal extent of the particle initially located at the inner edge of the core in lower density cases. Three different beam densities are considered, i.e., (a) $\eta_{x}=0.98$, (b) $\eta_{x}=0.95$, and (c) $\eta_{x}=0.92$. Other parameters are fixed to $M=0.13$ and $\xi=0.15$.
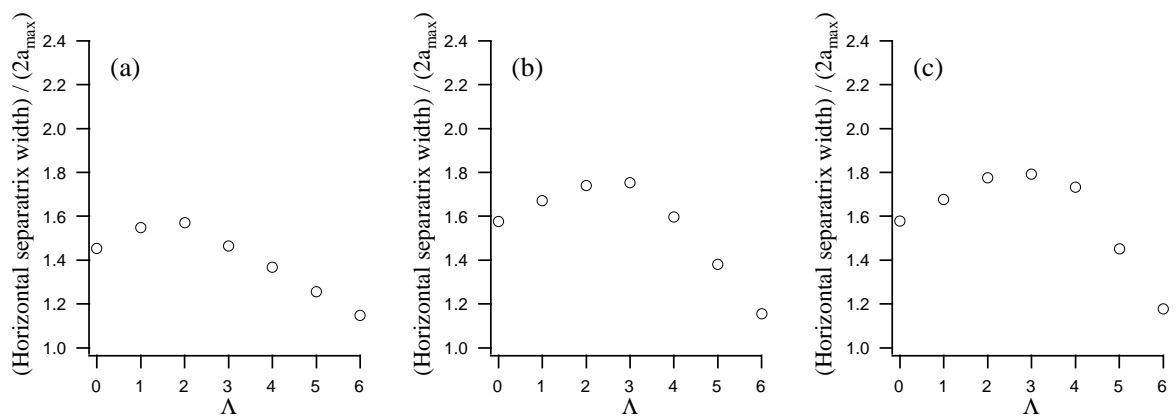

FIG. 8. The horizontal separatrix width of the 2:1 particle-core resonance in lower density cases. Three different beam densities are considered, i.e., (a) $\eta_{x}=0.98$, (b) $\eta_{x}=0.95$, and (c) $\eta_{x}=0.92$. Other parameters are fixed to $M=0.13$ and $\xi=0.15$.

width and halo intensity, and more precise dispersion matching is needed in cases with large $\Lambda_{\max } / \Lambda_{0}$. The halo width can be reduced by dispersion matching to roughly the same level as in the case of $\Lambda_{\max } / \Lambda_{0}<1$. In conclusion, it is advantageous to make $\Lambda_{\max } / \Lambda_{0}$ small to reduce both the width and the intensity of halos. Small $\Lambda_{\max }$ is achieved if the longitudinal beam profile is sharp edged. It is efficient to reduce $\xi$ to make $\Lambda_{0}$ large, which can be achieved by, for example, adopting a lattice structure with large bare tune. These results provide a practical criterion in designing high-intensity circular accelerators from the viewpoint of beam loss minimization.

\section{B. High density cases}

Although this particle-core model is constructed assuming modest density beams, it is applicable to high density cases provided that $\xi$ is small. As increasing beam density is required in future applications, it seems worthwhile to pursue halo dynamics in high density rings based on this particle-core model, while we should keep in mind 

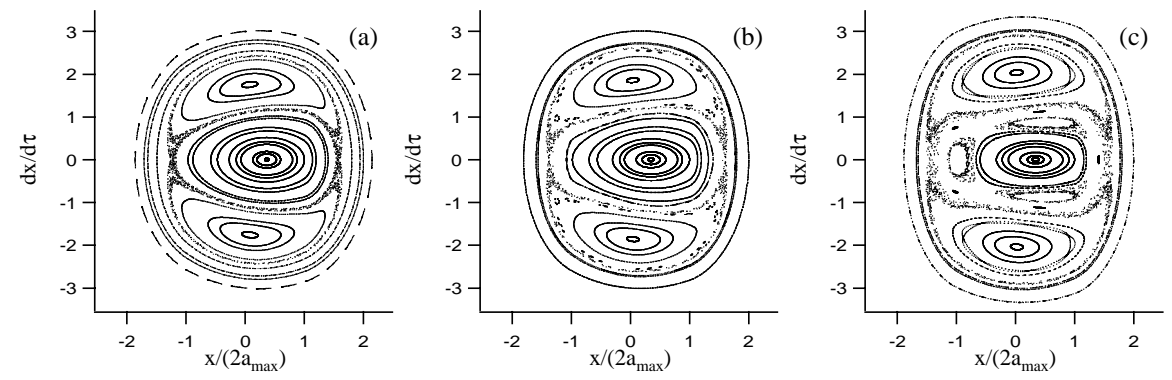

FIG. 9. The Poincaré surface of section plots for test particles in high density cases. The test particle position scaled by the maximum beam boundary width $2 a_{\max }=2 a(0)$ is taken as the abscissa. Beams with three different densities, i.e., (a) $\eta_{x}=0.5$, (b) $\eta_{x}=0.4$, and (c) $\eta_{x}=0.3$, are considered. Other parameters are fixed to $M=0.10, \Lambda=4$, and $\xi=0.15$.

that high density rings often have large $\xi$. Poincaré plots for high density beams are shown in Fig. 9, where three different beam densities have been assumed. In increasing beam density keeping $\xi$ to 0.15 , the island surrounding the central stable point is distorted and some additional islands of higher order resonances appear around that. Because of the overlap of these higher order resonances, weak chaosity is observed in all three cases in Fig. 9, which may increase halo intensity.

\section{SUMMARY}

We have constructed a simple particle-core model for beams in circular accelerators, neglecting fourth or higher order contribution of $\xi$, which is a parameter we introduce as a measure of the strength of dispersion effect. Although this model is efficient only in cases where $\xi$ is small, it is applicable to a large class of circular accelerators designed for modest-density applications and it provides us with useful information on halo dynamics in these machines. This model is especially sufficient for halo studies in most rings designed as a bunch compressor for spallation neutron sources where $\xi^{2}$ is typically around or smaller than 0.1 .

This model is applied to isotropic beams in a smooth ring in order to investigate dispersion effect on halo generation considering the cases where breathing mode of core oscillation is selectively excited. In systematic numerical analyses, it is found that the presence of dispersion simply gives a shift of fixed point locations in modest density cases with $\eta_{x} \geq 0.9$, causing no chaotic motion of test particles. The shift enables some of the test particles to orbit outside the separatrix of a 2:1 resonance, which is in striking contrast to cases without dispersion where particles are bounded by the separatrix. Dispersion matching is essential to suppress this diffusion if $\Lambda_{\max }$ is larger than a certain threshold $\Lambda_{0}$. As $\Lambda_{0}$ varies roughly inversely as $\xi$, rings with small $\xi$ have an advantage in preventing the diffusion due to dispersion mismatch.

In cases where the diffusion is suppressed, halos are formed by the same mechanism as in a straight channel, namely, the 2:1 particle-core resonance. The width of halos formed by the particle-core resonance is found to be about 1.6 to 1.8 times the maximum core width in typical rings for modest density applications, which is a little narrower than that in a straight channel where the ratio of the halo width to maximum core width is typically two. These results give us a practical criterion in determining physical aperture and choosing the lattice structure of high-intensity circular accelerators.

As seen in this paper, the particle-core analysis provides us with a clear physical insight into halo dynamics and gives us some practical information on halos in circular accelerators. However, it is important to bear in mind that the particle-core analysis is unable to predict the rate of halo formation. Obviously, it is an important issue in ring design whether a halo is developed in its cycle from injection to extraction. Although the rate of halo formation is known to be slower with lower beam density, there seems to be a good possibility of developing halos in the typical cycle from injection to extraction of modestdensity circular accelerators taking into account that path length in a ring is much longer than in a linac. To confirm this expectation, self-consistent simulations employing macroparticles are necessary. For circular accelerators, only a few attempts have been made to examine halo properties using self-consistent macroparticle simulations $[3,5]$, which is in a striking contrast to straight channels where extensive particle-in-cell (PIC) simulations have been performed [2]. More extensive PIC simulations are necessary to confirm findings obtained by the particle-core analysis and deepen our understanding of halo dynamics in circular accelerators.

[1] J.S. O'Connel, T.P. Wangler, R.S. Mills, and K.R. Crandall, in Proceedings of the 1993 Particle Accelerator Conference, Washington, DC, edited by S. T. Corneliussen (IEEE, Piscataway, NJ, 1993), p. 3926; J. M. Lagniel, Nucl. Instrum. Methods Phys. Res., Sect. A 345, 46 (1994); 345, 405 (1994); R.L. Gluckstern, Phys. Rev. Lett. 73, 1247 (1994); A. Riabko, M. Ellison, X. Kang, S. Y. Lee, D. Li, J. Y. Liu, X. Pei, and L. Wang, Phys. Rev. E 51, 3529 (1995); Q. Qian, R.C. Davidson, and 
C. Chen, ibid. 51, R5216 (1995); Y. Fink, C. Chen, and W. P. Marable, ibid. 55, 7557 (1997); T. P. Wangler, K. R. Crandall, R. Ryne, and T. S. Wang, Phys. Rev. ST Accel. Beams 1, 084201 (1998); M. Ikegami, Phys. Rev. E 59, 2330 (1999).

[2] R. A. Jameson, in Proceedings of the Joint US-CERNJapan Accelerator School 1994 (World Scientific, Singapore, 1996), p. 530; Los Alamos National Laboratory Report No. LA-UR-93-1209, 1993 (unpublished); H. Okamoto and M. Ikegami, Phys. Rev. E 55, 4694 (1997); M. Ikegami and H. Okamoto, Jpn. J. Appl. Phys. 36, 7028 (1997).

[3] S. Machida and M. Ikegami, in Workshop on Space Charge Physics in High Intensity Hadron Rings, edited by A.U. Luccio and W.T. Weng, AIP Conf. Proc. No. 448 (AIP, New York, 1998), p. 73.

[4] J. A. Holmes, J.D. Galambos, D. K. Olsen, and S. Y. Lee, in Workshop on Space Charge Physics in High Intensity Hadron Rings (Ref. [3]), p. 254; in Proceedings of the Sixth European Particle Accelerator Conference, Stockholm, 1998 (IOP, London, 1998), p. 279.

[5] J. A. Holmes, J. D. Galambos, D. K. Olsen, J. H. Whealton, M. Blaskiewicz, A. Luccio, and J. Beebe-Wang, in Proceedings of the Sixth European Particle Accelerator Conference, Stockholm, 1998 (Ref. [4]), p. 1109.

[6] M. Ikegami, Nucl. Instrum. Methods Phys. Res., Sect. A 435, 284 (1999).

[7] M. Reiser, S. Bernal, A. Dragt, M. Venturini, J. G. Wang, H. Onishi, and T.F. Godlove, Fusion Eng. Des. 32, 293 (1996).

[8] M. Kinsho, J. Kusano, M. Mizumoto, and F. Noda, in Proceedings of the Sixth European Particle Accelerator Conference, Stockholm, 1998 (Ref. [4]), p. 844.

[9] C. J. Gardner, Y. Y. Lee, and A. U. Luccio, in Proceedings of the 1997 Particle Accelerator Conference, Vancouver, Canada (IEEE, Piscataway, NJ, 1998), p. 962; I. S. K. Gardner, H. Lengeler, K. Bongardt, H. Klein, G. H. Rees, and C. W. Warsop, ibid., p. 988.

[10] T. Uesugi, S. Machida, and M. Ikegami, in Proceedings of the 1999 Particle Accelerator Conference, New York (IEEE, Piscataway, NJ, 1999), p. 1821.

[11] We have adopted envelope equations derived in M. Venturini and M. Reiser, Phys. Rev. Lett. 81, 96 (1998) as the starting point of our analysis, while an alternative form of envelope equations is derived in $\mathrm{S}$. Y. Lee and H. Okamoto, Phys. Rev. Lett. 80, 5133 (1998).

[12] I. M. Kapchinskij and V. V. Vladimirskij, in Proceedings of International Conference for High Energy Accelerators, Geneva (CERN, Geneva, 1959), p. 274.

[13] See, for example, O.D. Kellog, Foundation of Potential Theory (Ungar, New York, 1953), Chap. IV.

[14] Recently, NSP was reviewed and a new joint project based on NSP of JAERI and Japan Hadron Facility project of KEK has been proposed. In the joint project, a $1 \mathrm{MW}$ rapid cycling synchrotron is adopted for bunch compression in the first phase, and using two or three storage rings is considered as an option for the second phase to upgrade the total beam power to $5 \mathrm{MW}$. In this paper, we take parameters of the original NSP ring as an example, because the detailed design of rings for the joint project has not been fixed at the present time. For the original design of the NSP ring, see Ref. [8]. For the outline of the joint project, see The Joint Project Team of JAERI and KEK, Japan Atomic Energy Research Institute Report No. JAERI-Tech 99-056, 1999 (unpublished).

[15] E. Forest and R. D. Ruth, Physica (Amsterdam) 43D, 105 (1990); H. Yoshida, Phys. Lett. A 150, 262 (1990). 